of in hot or muggy weather. ${ }^{10}$ Further, boric acid is very widely used commercially; it may legally be added to cream and butter; it has often been found in tinned foods, dried fish, and meats, bottled (liquid) eggs, \&c. A patient may therefore be poisoned by one or more of a variety of comestibles.

\section{SOME REMARKS ON}

\section{"SPANISH INFLUENZA" :}

\section{ITS NATURE AND FTIOLOGY.}

By ALEXANDER EDINGTON, M.D., F.R.S. EDIN.,

MEDICAL SUPERINTENDENT OF GREY'S FOSPITAI, PIETERMARITZBURG LATE LIEUTENANT-COLONEL, S.A.M.C., E.A. FXPIEDITIONARY FORCE ; FORMERLY DIRECTOR OF THE BACTEKIOLOGICAC INSTITUTE, CAPE COLONY.

I Took over charge of Grey's Hospital in January, 1918, and admitted cases of influenza every month from that time onward. In the majority of cases the malady was uncomplicated, but in some there followed a true influenzal pneumonia of the type so common in the Edinburgh epidemic of 1889. Only one case died up to October, 1918, but about the 12th of that month there was an enormous rush of cases, and between that date and the end of the month 234 cases were admitted, of which 41 died (29 natives and 12 whites). At that time, owing to the war, I was the only resident, and owing to the amount of work in the town and district the visiting staff were unable to render any assistarice.

In the 1889 epidemic there was no heavy mortality, although, following in its wake, we had a large mortality from other diseases, especially tuberculosis, which had been lit up, perhaps, by the influenza, or, at least, had profited by the lowered resistance of its victims. In the case of the socalled "Spanish influenza," however, the mortality has been so enormous as to raise a grave doubt as to whether this mortality was actually attributable to influenza or to some other more malignant infection. If it is a new disease, what are its characteristics, or, if not, is it a recrudescence of some malady which has lain dormant for some generations?

\section{Symptoms of the Epidemic.}

The main symptoms of this epidemic in my experience, have been :-

(a) Malaise, headache, and pains in the body. In the fatal cases pains extending down whole length of the back have been common.

(b) The temperature has usually risen rapidly, but there has rarely been a true hyperpyrexia. Where this has occurred it has usually been within the first few days and, I think, was due to true influenza. But in certain fatal cases the temperature fell about the fourth or fifth day, and rose again about the fifth or sixth day, presenting a very different curve. I consider that in such cases the first curve was true influenza arising after a short period of incubation, and that the second curve arose after a longer incubation and was due to an unknown factor.

(a) The pulse rises with the fever, but during the curve of the second stage there is a very definite rise in blood pressure, and the pulse is hard and bounding.

(d) Following upon the rise in blood pressure there is frequently a short explosive cough when gelatinnus masses of mucus, and sometimes clots of blood, are forcibly ejected. In one case there was an actual rupture of a blood-vessel attended with profuse bæmorrhage.

(e) The delirium comes on about the third night, and is not severe at first; the patient can be roused into a lucid interval; but after about 48 hours the delirium is much more severe. and lucid intervals are rarer. Eventually it is of a violent character and there is difficulty in keeping the patients in bed.

(f) In the cases which went on to a fatal termination there has usually been an entire absence of any definite pathological symptom in the langs until crackling sounds are heard. This symptom usually comes on very late in the illness, and foreshadows death. The sound is very peculiar, and is such as one might expect if the lungs had been removed and the thorax packed with dry, crushed paper.

10 Its ase at all in milk is, of course, now illegal in this country.
But even after this crackling has been manifested for some time there is little or no alteration in the percussion note.

(g) The last symptom is one of the most seriou importnamely, the blackening of the nails and the dusky hue of the face. This has been commonly the forerunner of death, and is so characteristic that the public have taken notice of it.

\section{Post-mortem Examinations.}

I regret that I have not had the opportunity of seeing as many post-mortem examinations as I could have wished, and I am much indebted to my friend Mr. C. Ward, the district surgeon, for assisting me in making examinations of my own cases and letting me have access to all that he could. In view of the preceding crackling sounds one was led to expect great changes in the lungs, but there is little actually to be found. Usually there is some excess of pleural and pericardial fluid; the lungs are deeply engorged and, when cut across, a dark fluid of the consistence of glycerine exudes. There is no pneumonia, either lobar or bronchial; the lungs are quite buoyant and float in water. In my opinion the fatal issue, so far as the lungs are concerned, is due to a thrombosis of the whole of the pulmonary vessels. The vessels of the meninges are also congested and we have taken long thrombi from them. Of the abdominal organs the liver and kidneys are usually very deeply congested, but the spleen is not usually enlarged although it may be soft and friable.

\section{Basteriological Investigation.}

I did not attempt a thorough investigation since I had no laboratory to work in and did not really have the time to spare, but I shall state briefly such observations as I was able to make.

Examination of the scanty sputa of the delirious cases showed, in somes cases, the presence of bacilli of the plague type, but not absolutely typical. The bi-polar staining was not so clearly nor so sharply defined as in plague. An injection of a few drops of such a sputum into the subcutaneous tissue of a guinea-pig was followed by its death in six days, but it did not show any illness until the day previous to its death. An examination of its spleen showed the presence of small bacilli which gave an incomplete bi-polar staining. They frequently appeared in short chains of two or three, and might easily be mistaken for streptococci at a first glance. A gelatinous membrane surrounded them. I did not make pure cultures. Subsequent to this, examination of spleen smears from fatal human cases showed the presence of similar bacilli, but it was remarkable that they were never present in great numbers and appeared to be irregularly distributed in the organ. I succeeded in nbtaining pure cultures from a series of five cases. More recently several peculiar cases have been seen where there were no suspicion of any influenza and the symptoms were fever with delirium passing quickly into unconsciousness. In one of them there were peculiar nervous convalsive movements of the arms. At the post-mortem of this case we found the lungs quite normal; the spleen was not at all enlarged and was unfortunately not examined. On opening the skull there was found a small patch of grey exudate of a somewhat dry granular character, a smear of which was negative. The ventricles were distended with fluid. I opened the left ventricle of the brain aseptically and spread an $\ddot{0} \cdot$ eful of the fluid over a sloped agar tube. Eight pure colonies of the same bacillus, that I had already isolated from the spleens, were found.

In smears of the spleen containing this bacillus one finds many oval bodies showing bi-polar staining, having a strong similarity to the bacilli of bubonic plague. In size they vary from 1.0 micron to 1.75 micron in length and 0.5 to 0.75 micron in breadth. But one also finds somewhat amorphous bodies (these occurred largely in the first spleen I examined, but I failed to identify them as being organismal). These are but an exaggerated example of those found in bubonic plague.

Inoculation of this culture subcutaneonsly into a guinea-pig was followed by death six days later. There was a considerable amount of a greyish hæmorrhagic œdema at the site of the injection, a smear was found to be crowded with the characteristic bacilli and ovoid forms. The peritoneum was practically devoid of fluid. The liver was much congested, but the spleen was very little, if at all, enlarged and was of a ruby colour. Intra-peritoneal injection into a guinea-pig was followed by death in 48 hours. The peritoneal fluid was swarming with the bacilli, of which 
some had formed rather longer filaments. Pure cultures were obtained directly from this fluid. There was a remarkable pleuritis consisting of the greyish exudate which, however, was not at all like pus. This material was filled with the bacilli, a smear of which might almost be mistaken for a smear of a bubonic plague spleen.

The bacillus grows rapidly in bouillon and possesses a very disagreeable odour. (Patients in an advanced state of the disease also have a very peculiar odour which is difficult to describe, but to my mind is sour and musty.) On agar it does not form a very characteristic growth and it is interesting to note that different samples show slight differences. The first culture was obtained under difficulties and was incubated for some time at a higher heat than I wanted. It shows a free, sticky, greyish-white growth. After passing through the peritoneum of a guinea-pig the culture was undoubtedly changed, in that the glutinous character was much less evident, although the morphology was still the same.

I have made a series of attempts to isolate the bacillus from the blood during life, but have never succeeded. In the case of the bacilli obtained from the left ventricle of the brain the fluid was drawn within three-quarters of an hour after the death of the patient.

I should mention that the guinea-pig inoculated intraperitoneally showed violent convulsive movements of the body for some hours previous to its death. The others inoculated subcutaneously showed no such movements, but passed into a lethargic condition ending in death.

One cannot say that the bacillus which has been isolated is actually the cause of the disease, although the fact of finding it so often and the results obtained by inoculation are certainly very suggestive. If it is the cause it is worthy of note that the virulence very soon disappears in artificial cultures.

It is one of the most pleomorphic organisms which $I$ have ever had to deal with, following very much the line of bubonic plague bacilli. When grown in bjuillon for six hours, examination after staining with carbol-methylene blue shows bacilli with rounded ends clearly giving a bi-polar staining. On agar the growth is that of bacilli mostly, and after a few days' growth there is great evidence of degeneration of the bacilli. One finds many of them with ends looking as if irregularly broken off. I have never seen such a peculiar condition before in any bacillus with which $I$ have had to deal. The growth on ayar is at first fairly profuse, but if removed from the incubator and allowed to grow at the ordinary room temperature one finds a secondary growth taking place. This is much thinner than the original, and projects away from the main growth in a thin plate made up of irregular strim running out at right angles to the growth and granular in appearance.

It is extremely interesting to note that the spleen must carry much more bacilli than microscopical examination would make one expect. On the examination of one spleen I had difficulty in seeing bacilli and had to search numerous fields to find sufficient to justify an assertion that they were actually there; but on touching the spleen pulp with an öse and rubbing this over a sloped agar tube I obtained a nearly confluent growth. I had the pleasure of demonstrating this spleen smear and the tube of colonies to the Influenza Epidemic Commission on their vlsit to this hospital.

I have satisfied myself that the malady is a definite infective disease of high virulence. Indeed, in my opinion, it has probably accounted for the most of the death-rate of the so-called influenza in this province. I cannot, however, suggest the manner of its spread. I have not seen a case arising from direct infection; although a heavy percentage of the nurses went down it was from simple uncomplicated influenza from which they all recovered. The cases can be diagnosed very soon after admission, in some cases, especially if there are no lung symptoms and the temperature is not over $104^{\circ}$, and if the nervous condition is well marked. Oases in which a moderately high temperature remains high for 48 hours without lung symptoms are to be regarded with suspicion. The third type, which is not so common in the present epidemic as it was last October, is that in which the patient is admitted with a rising temperature which forms a curve extending over about five days, drops to nearly or quite normal, and is then followed by a sharp rise and remains high.

Treatment.

As regards treatment, so far as my experience goes, nothing seems of great avail. Recently we have been attempting to treat the delirium as early as possible with sedatives. Of these ammonium bromide is peculiarly beneficial. Under a dosage of $10 \mathrm{gr}$. per hour the delirium is somewhat abated and the pulse softens, but where it is insufficient paraldehyde, chlosalamine, or hyoscine may be used. I venture to suggest that morphia is contra-indicated as tending rapidly to permit pulmonary cedema, which is the forerunner of a fatal issue. This treatment has undoubtedly the resulo of delaying the fatal issue for several days. In such cases one is able to note the enormously increased reflexes. These are so well marked that it is almost impossible to take the pulse at the wrist.

The disease would seem to be one affecting the cerebrospinal nervous system, and to be more irritative than inflammatory. Pulmonary and other conditions which may supervene might be regarded only as complications, arising from the disorganisation of the nervous system.

\section{INFLUENZAL BRONCHO-PNEUMONIA AND PNEUMONIA TREATED WITH THE ARMY MIXED VACCINE.}

FEBRUARY TO MAY, 1919.

BY J. BLACK.MILNE, M.B. ABerd., AND

KENNETH ROGERS, O B.E., M.D. LOND., PHXSICLANS TO BROOK WAR HOSPITAL, WOOLWICH, S.E.

BEFORE dealing with our experience of the vaccine treatment of influenzal pneumonias, we will first give the number of cases of influenza ( 3432 in all) and the death.rate, in the three waves, so far as this hospital was concerned: May to August, 1918, admitted 1809 cases with 10 deaths ; September to December, 1918, admitted 1131 cases with 38 deaths ; January to May, 1919, admitted 492 cases with 14 deaths. In the first two periods no vaccine was used.

The accompanying series of eight charts are taken from 35 similar chtrts, carefully recorded, of cases of bronchopneumonia and pneumonia treated by vaccine here.

\section{Clinical Notes of Eight Cases.}

Case 1.-The main features of this case were the marked cyanosis and prostration of the patient. Râles were heard all over both lower lobes, and the sputum was red-brown. Four injections were given in all, the dose increasing by m. i. from m. iii. to m. vi., and the patient made a rapid recovery.

CASE 2.-Râles with a similar distribution were heard in this patient; the sputum was copious and red for five days. One injection, m. iv., was given.

CASE 3.-This patient was cyanosed and had herpes on lips, besides râles in both lower lobes and red-brown sputum. Two injections, m. iii. and m. iv., were given, and he made a rapid recovery.

CASE 4.-In this case there was massive consolidation in the right lung, as well as râles in both lower lobes; the patient was cyanosed; he made a gradual recovery, with prostration. One injection only, m. ili., was given.

CASE 5.-This was a case of pneumonia of the lobar type, with red-brown sputum and massive consolidation in the right lower lobe. Two injections, m. v. and m. viii., were given on the third and the fifth days of illness.

CASE 6.-Epistaxis was a feature of this case, which was of the broncho-pneumonic type. An injection of m.iii. was given on the seventh day, when the temperature rose to $104.8^{\circ} \mathrm{F}$., pulse 80 , respirations 44 , and $\mathrm{m}$. v. on the following day. The right base was dull, and the patient showed the same conditions as to râles in lower lobes, prostration, cyanosis, and red-brown sputum as the other cases; he made a slow revovery.

CASE 7.-In this case there was marked dusky violet cyanosis to the tenth day, and also consolidation of the red lower lobe. Râles were heard all over the chest, and the sputum was brown. The patient showed marked drowsiness till the seventh day, and recovered gradually after the tenth day. Two injections of $\mathrm{m}$.iii. and one of $\mathrm{m}$. $v$. were given in all.

CASE 8.-This was a case of very severe broncho-pneumonia with slow recovery. Four injections were given, as in Case 1. The cyanosis, violet at first, dusky later, lasted for 14 days; the patient was drowsy and had tremors. There were râles over both lower lobes. On the eleventh day of illness the respirations were still 44 , pulse 108, though the temperature was normal. 